\title{
Priority Research themes in the fight Against the COVID-19 with particular reference to Cameroon *
}

\section{Vincent PK Titanji, PhD}

Cameroon Christian University Institute, PO Box 5, Bali, North West Region, Cameroon

Address for correspondence: vpk.titanji@yahoo.com

\begin{abstract}
The ongoing SARS CoV-2 pandemic also known as COVID-19 is a highly infectious and deadly disease that has disrupted socio- economic activities and killed over 500000 people worldwide during the past six months since it first erupted in Wuhan China in December 2019. While intensive efforts are under way in the developed countries to find a vaccine and cure for the disease, Cameroon and other African countries should not fold their hands and wait, but join the search for new remedies including from cures from traditional herbs while rigorously applying barrier and social distancing measures that have proven effective in in curbing the spread of the disease. Herein we enumerate a short list of research priorities that are feasible in our milieu and that could improve on diagnostics, treatment and prevention of the disease in the short and medium terms.
\end{abstract}

Key Words: SARS CoV-2, COVID-19, research, diagnostics, medicinal plants, traditional medicine, pandemic, Cameroon.

\section{Resume}

La pandémie en cours du SRAS CoV-2, également connue sous le nom de COVID-19, est une maladie hautement infectieuse et mortelle qui a perturbé les activités socio-économiques et tué plus de 500000 personnes dans le monde au cours des six derniers mois depuis son apparition à Wuhan en Chine en décembre 2019. Alors que des efforts intensifs sont en cours dans les pays développés pour trouver un vaccin et guérir la maladie, le Cameroun et les autres pays africains ne devraient pas croiser les mains et attendre, mais se joindre à la recherche de nouveaux remèdes, y compris à partir de remèdes à base d'herbes traditionnelles, tout en appliquant rigoureusement une barrière et des mesures de distanciation sociale qui se sont révélées efficaces pour enrayer la propagation de la maladie. Nous énumérons ici une courte liste de priorités de recherche réalisables dans notre milieu et susceptibles d'améliorer le diagnostic, le traitement et la prévention de la maladie à court et moyen termes. ( Crédit : Google Translator.)

Received: 10/06/2020

Accepted: 10/07/2020

DOI: https//dx.doi.org/10.4314/jcas.v15i3.5

(C) The Authors. This work is licensed under the Creative Commons Attribution 4.0 International Licence. 


\section{INTRODUCTION}

The Corona Virus Disease (COVID-19) has been making headline news since December 2019 when it first broke out in Wuhan, China. We witnessed with horror how it spread in Europe, then North America, particularly the USA, holding our breaths and wishing that this time around Africa would be spared. This hope was short lived as the African continent soon became affected with new infections and death counts now rapidly rising in many countries. Today the question is how to stop the spread of the disease, treat those who are infected and prevent future infections. These questions are classical in the sense that they apply to all epidemics. They are posed with increasing urgency because SARS $\mathrm{CoV}-2$, the virus that causes COVID-19 is highly infectious, is at least $10-20$ times more lethal than the common cold, and does not respect socio-political and economical boundaries (WHO,2020). The World Health Organization (WHO) The African Academy of Sciences (AAS) have defined urgent research priorities to address the COVID19 pandemic. Recently, a group of researchers in a thought-provoking editorial entitled, Shining the Light over Africa, defined urgent actions to be taken against the pandemic (Rosenthal et al; 2020). In this brief report, I focus on those research topics which are relevant to the Cameroonian context and by extension to subSaharan Africa. For greater impact I have chosen a question and answer format to introduce the priorities. I then expand on the prerequisites for working with highly infectious pathogens like SARS CoV-2 especially in resource constrained countries like ours. I conclude with perspectives and future directions.

\section{How do we stop the spread of COVID-19?}

The answer to the first question is known. Isolate the infected persons from the rest of the population and institute barrier and distancing methods such as have been described by the
WHO and public health agencies around the globe. These strategies are time honored and have been shown to be effective in stopping the spread of recent epidemics and pandemics like Ebola in the DRC and in West Africa and the ongoing COVID-19 in countries like Singapore and South Korea, etc. However, we have witnessed how difficult it has been to implement effective quarantine measures through lockdowns and stay home injunctions without totally destroying the economy and normal social life.

The relevant question is how to adapt effective infection control strategies to our context so that life can continue more or less normally while seeking long-term solutions to address this pandemic. The Academy can provide leadership through its college of social sciences to provide guidance on effective communication and sensitization methods to effectively enforce the quarantine strategies.

\section{How do we design clinical studies to evaluate therapies for COVID-19 adapted to our context?}

The question of evaluating effective treatments for the disease is equally high on the agenda. There are currently limited treatment options for COVID-19. An NIH led randomized clinical trial recently showed that the antiviral drug Remdesivir provides modest benefit by reducing the time to recovery by $33 \%$ for patients with severe COVID19 when compared to placebo. Although now available in the USA through an emergency use authorization (EUA) this drug has not been evaluated in the African context and is currently not available to our populations. Supportive care measures for patients with COVID-19 remains the mainstay of treatment for patients in Cameroon, but our healthcare systems have limited capacity to provide a high level of care such as mechanical ventilation and other critical care interventions. Therefore, innovations adapted to the African context are encouraged to provide supportive care 
to those who are infected and require hospitalization. In this regard the invention of a cost-effective ventilator in Senegal should be encouraged.

More than 100 drugs are under various stages of clinical development in the developed world, particularly in China, USA and Europe, and it is hoped that once a treatment is found it will eventually get to Africa. This hope, however, should not discourage local efforts to find new treatments for the disease. Even when drugs are discovered in the more developed countries, it takes time and significant financial investment to make these interventions available to the African population. The case of anti-retroviral drugs for HIV reminds us of the precarity of depending entirely on externally sources of medication supply whilst neglecting local possibilities. The medicinal plants of Africa are a rich resource that have historically produced cures for many endemic diseases. The announcement by Bishop Kledar of Cameroon and the President of Madagascar of herbal treatments for COVID19 are encouraging signs that African traditional medicine should not be neglected in the search for cures for COVID19.

However, two important conditions must be met by all drugs destined for human consumption: their safety and efficacy must be demonstrated through ethically conducted research studies. This last requirement has not been formalized in Cameroon, where traditional healers produce and distribute their preparations without licensing or registration. The Academy can provide guidance on the testing of traditional medicine preparations for the treatment of fevers for possible activity against corona viruses. It should be pointed out that plants are rich sources of anti-viral agents, some of them falling in the chemical categories of essential oils that might be of generated in herbal steam- bath treatment of fevers.

Several projects can be initiated in the broad category of drug development. This is, however, a long and expensive process from discovery, pre- clinical studies, clinical trials, registration, manufacturing and distribution to pharmaco-vigilance. The process is well codified and regulated in countries around the world, but will be challenging to establish in the timeframe needed to address the pressing outbreak of COVID-19 in Cameroon.

For traditional medicine preparations the process may be simplified, without compromising safety and efficacy (WHO,2000). This then gives an opening for the Academy to develop projects aimed at testing and validating traditional medicine preparations following the scientific method. The Academy may also develop projects aimed at validating antivirals isolated from plants, first in vitro, and subsequently in animal models thus following the classical pathway for drug development. (Reichling et al; Forsch Komplementmed, 16(2):79-90;2009) There is currently a lot of concern on social media that Africans will be targeted as "guinea pigs" for drug and vaccine development. International collaboration is crucial in the fight against the COVID19 and African countries cannot apriori exclude themselves from participating in the research for solutions. What needs to be done is to ensure that the same ethical and safety standards are respected for research in evaluating new drugs and vaccines. For this to happen African countries need to build expertise in all aspects of drug development including the ability to verify on a case by case basis all medications and vaccines. We already have such a structure in many African countries including Cameroon, but these need to be strengthened. The Academy can take the lead in strengthening through training 
and monitoring our drug and vaccine experimentation and registration platforms.

\section{How can COVID19 be prevented?}

Methods for disease prevention are many and varied depending upon the particular ailment, its causes, and means of transmission. For transmissible diseases and some forms of cancer vaccines are a major and particularly effective means of prevention. With regards to COVID19 more than a 100 vaccine trials are ongoing, with the expectation that a vaccine could become available in 12-18 months. Vaccine development is similar to drug development in many aspects though even more rigorous than drug development in some respects. Though we can be cautiously optimistic in our hope for and effective vaccine, SARS-COV-2 is a virus that is newly introduced into the human species and our understanding of the pathogen and the disease need to improve before a vaccine can be developed. Of note MERS-CoV and SARS-CoV1 are similar to SARS-CoV2 and have been around much longer and we still do not have effective vaccines for these.

Many of the vaccine candidates in clinical trials are based on the 'golden bullet' principle whereby the vaccine is designed to block a single targeta kind of heal of Achilles-thereby neutralizing the pathogen. Such univariate vaccines work best when the target does not mutate frequently, and where the function to be neutralized or blocked is not by-passed by other mechanisms. Without digressing into the theories of vaccination, let me say that we have challenged the golden bullet or univariate target approach at least in the case of Plasmodium falciparum malaria, where we have used a multivariate approach to develop a malaria vaccine candidate that works well in mice, but remains to be tested in humans (Titanji et al, 2017 \& 2009; Dinga et al,2018).

Vaccine development therefore offers another opportunity for the Academy to intervene with projects, including:
- the investigation of acquired immunity to SARS-CoV-2 in our milieu;

- determining whether the antibodies developed are neutralizing and therefore protective;

- designing and testing multivariate vaccines, at least in vitro, before moving on to the other steps in vaccine development pipeline.

What are the prerequisites for working with highly infectious agents like SARS CoV-2, the causative agent of COVID?

The corona virus is a very highly infectious pathogen. Specialized training and specialized laboratories are required to work with this virus. At least a Level-3 containment laboratory is required to work with the intact virus whereas growing the virus in cell culture requires Level-4 containment. To my knowledge the most sophisticated laboratories in Cameroon are Level2 containment laboratories. The Biotechnology law of Cameroon makes mention of these levels of containment and special authorizations are required to create level-3 and Level-4 laboratories. The Academy can provide advice and training in collaboration with the Ministry of Research and Innovation on the setting up, regulation, and functioning of Levels $3 \& 4$ Containment laboratories. Appropriate infrastructure is required to carry out research safely on COVID19 and other infectious pathogens. In the short term I recommend that the Biotechnology Center in Nkolbisson Yaoundé and the Biotechnology Unit at the University of Buea be strengthened with Level-3 laboratories since they have both the space and the expertise for work with infectious organisms.

\section{Urgent themes of Research.}

Considering what has been stated above I propose that the Academy includes the following eight themes among its priorities for fighting COVID-19.

6.1 Characterization of the virus (SARS CoV-2) the causative agent of COVID19. It is 
important to isolate and sequence the genomes of the COVID 19 circulating in our milieu. This can be done within a national or international collaborative effort. Access to reliable genomic data is indispensable for development of diagnostics, therapeutics and vaccines.

6.2 Diagnostics. New rapid tests that can be performed in resource poor environments at affordable costs are needed.

6.3 New drugs: Our Focus should include working with plant derived medicines since plants have proven to be reliable source of new drugs.

6.4 Vaccines and vaccination: A better understanding of the immune responses to SARS CoV-2 in our milieu is necessary for both evaluation of new vaccines, and design of immunotherapies.

6.5 Epidemiology. An understanding of the spread of the disease, its transmission modes, and its disaggregated prevalence in the population important for rational control strategies.

6.6 Mathematical modeling tools should be developed/adapted to enable the follow up and prediction of disease trends in a bid to better deploy control measures.

\subsection{Human behavior and response to the} pandemic. Knowledge attitudes and Practices (KAP) studies should be undertaken in our communities about the COVID-19 its spread and management. This topic is being addressed by the College of Social Sciences to which the reader is referred.

6.8 Capacity building at the level of infrastructure and technical competence is indispensable for achieving themes 1-7 above.

\section{Conclusions}

It should be emphasized that even if new drugs and vaccines are approved within the time limit predicted, it will take time to reach end users in Africa. In the meantime, the infection control strategies have proven their efficacy in controlling the spread of infectious disease pathogens, and as such, should be rigorously applied.
- An ealier version of this review was submitted to the Cameroon Academy of Sciences Task Force of COVID-19 Statement.

\section{References}

Rosenthal, PJ;Breman,JG; Djimde, AA;John,C.C; Kamya,MR; Leke,RGF; Moeti,MR; Nkengasong,J; Bausch,D.G .(2020) Covid-19: Shining the Light on Africa. American Journal of Tropical Medicine and Hygiene pp1-4 doi:10.4269/ajtmh 20-0380

Muhammad Mukhtara a.d; Arshadb,M; Arshadb ,M; $\quad$ Pomerantzc,R.J; Wigdahl,B; Parveene,Z.(2008). Antiviral Potentials of Medicinal plants. Virus Research, 131: 111-120

Titanji,VPK; Dinga,NJ; Nyasa ,RB.(2017). A rational Approach for predicting the minimum composition of anti-parasite sub-unit Vaccines: A multiple target vaccine hypothesis. Journal of the Cameroon Academy of Sciences,14(1):3-10 Titanji, VPK; Amambua-Ngwa,A; Anong,DN; Mbandi,SK; Tangie,E;Tening,Ivo; Yengo,R Isolation and expression of UB05, a Plasmodium antigen recognized by antibodies from semiimmune adults in a high transmission endemic area of the Cameroonian rainforest. Clinical Chemistry and Laboratory Medicine,47(9):1147-1158; 2009; Dinga, JN; Gamua, SD; Ghogomu,SM; Titanji,VPK. (2018). Preclinical efficacy and immunogenicity to show that a chimeric Plasmodium falciparum UB05-09 antigen could be a malaria vaccine candidate. Parasite Immunology. 2018; e12514. wileyonlinelibrary.com/journal/pim | 1 of 9 https://doi.org/10.1111/pim.12514 WHO. (2020). Coronavirus Fact Sheet www.who.int.

WHO (2000). General Guidelines for Methodologies on Research and Evaluation of Traditional Medicine.WHO/EDM/TRM/ 2000.1 


\section{Instructions and Policy Information to Authors/Contributors}

\section{Introduction}

The Journal of the Cameroon Academy Sciences (JCAS) is a multidisciplinary publication of the Cameroon Academy of Sciences (CAS) that is devoted to all aspects of fundamental and applied re-search. It also publishes topical reviews on science and technology in development, arts, humanities and culture.

Opinions expressed in the journal are those of its authors and should not be construed as reflecting the official policy of the Academy. The journal will not publish articles dealing with political or religious advocacy. JCAS appears both online and in a print edition.

\section{Vision}

The Journal of the Cameroon Academy of Sciences aspires to become an authoritative source of scientific and scholarly information on the African Continent and worldwide.

\section{Strategic objectives 2019-2021}

The Academy has set the following objectives for JCAS over the years 2018 to2019:

- To revise the policy documents of the Journal to meet international standards and create a website for the Journal by the end of 2019;

- To ensure that JCAS appears regularly in at least three issues per year with each issue containing at least 5 articles.;

- To publish at least one special issue per year;

- To raise the IMPACT Factor of the journal above the median for African journals and towards a full digit;

- To update the Editorial Board by including more women and younger Scientists by the end of 2019;

- To raise enough funds from page charges and advertisements to enable the publication of the journal without subsidies.
Publication mode: From 2017 the JCAS has adopted the open access mode under the Creative Commons Attribution 4.0 International License ( https:// creativecommons.org/choose/ )

Archiving: The JCAS is archived by African Journals online (soft copy) ( http://www.inasp.info/ajol// ) and the Cameroon Academy of Sciences (printed edition). Henceforth all accepted articles will be assigned a DOI number before publication.

Language and style: Articles should be written in English or French and provided with abstracts in both languages. Articles should be written in the passive voice and in the third person. Pronouns such as "I" and "we" should be avoided except in cases where it is customary to do so.

Submission of articles: The manuscripts along with any display items such as tables, figures, photographs, etc. should be submitted online or by email. Hard copy submissions will not be accepted. Photographs should be in black and white. Colour photographs will only be published exceptionally and even then, at considerable extra cost to the author(s). The authors of accepted articles will be required to submit a final version of the manuscript on a CD/ROM or online using the software Microsoft Word or any other computer software recommended by the editor.

All submitting authors must counter-sign against their names on the title page to indicate their approval of the submission. The signed title page should be scanned and sent as an email attachment to the address given below.

The Editor-in-Chief, Journal of Cameroon Academy of Sciences, Biotechnology Unit, Faculty of Science, University of Buea, P.O. Box 63, Buea,

S.W. Region,

Cameroon 
Web site: www.ubuea.cm

Peer-review: All manuscripts submitted to the journal will be reviewed anonymously by three reviewers and will be accepted only when a majority of the reviewers recommend it for publication. Authors of rejected articles will be given an opportunity to reply to the reviewers' comments.

Page charges and offprints: Authors of accepted articles will be required to pay a fee of 20 USD or 10.000 FCFA per printed page. Contributors from Low Income Countries can apply for a complete or partial waiver of pages charges and will be given such if their articles are accepted. Contributors/authors will receive a pdf copy of their article from which they can make additional copies.

Persons interested in the print edition can place orders directly at the following prices:

120 USD for the Americas;

100 USD for Europe \& Asia

50 USD for Africa

and 30 USD for Cameroon per volume of three

issues. The price of postage by airmail is included in the rates given above.

Copyright: The submission of an article to the JCAS means that the same article is not being considered elsewhere for publication. Upon acceptance, the author(s) shall sign a declaration that they understand and accept that their article will be published in the Open Access mode under the Creative Commons Attribution 4.0 International License (https:// creativecommons.org/choose/)

\section{Plagiarism}

The JCAS does not tolerate plagiarism, defined as using other people's ideas without citing the authors. The responsibility of avoiding plagiarism resides on the author(s), while the Editor of JCAS reserves the right to conduct an electronic check of submitted articles. A plagiarized manuscript will be rejected outrightly. In the unlikely case that an article is found to have been plagiarized after its publication in the
JCAS, the article will be retracted and notification of retraction published in the next issue of the journal.

\section{Corrigenda}

In cases where an article has been published with material errors, the author(s) can petition the Editorin-Chief for a Corrigendum to be published, and if justified a corrigendum will be published in the next issue of the journal.

\section{Retraction}

If after publication an article is found to have been plagiarized, or to be based on fabricated data, the article shall be retracted or withdrawn. The initiative to retract an article shall lie with any of the authors and/or readers who shall file a petition with all necessary justifications to the Editor-in-Chief. The Editor-in-Chief shall act in consultation with the Editorial Board and with due diligence.

\section{Meta-analysis}

Review articles on randomized trials or evaluations must conform to the PRISMA (Preferred reporting items for systematic reviews and meta-analysis)

guidelines at http:// systematic

reviewsjournal.biomedcentral.com/10.1186/s13643017-05908)

\section{Preparation of Manuscripts}

Length of article: Manuscripts should be typed on A4 paper with double spacing and a $2 \mathrm{~cm}$ margin all round. A full-length article should not be less than 1000 and should not exceed 5,000 words. Commentaries and letters to the Editor should not exceed 1,000 words each.

Title: The title should not exceed 150 characters and should not contain abbreviations or acronyms. It should be precise and informative.

Title page: The title of the article should be typed on the first page of the manuscript followed by the names of the authors and the respective postal and email addresses of their institutions. The name and address of the corresponding author should be clearly identified in a foot-note. 
Organization of the article: Depending on the type of communication, the article should be divided into the following sections:

\section{Experimental Work}

- Abstract

-Introduction

- Materials and Methods

- Results

- Discussion

- Conclusions

- Acknowledgements

- References: Bibliography followed by webography

\section{Theoretical Work}

-Abstract

-Problem statement

-Approach, definitions, assumptions

-Solutions or proofs

-Discussion

-Conclusions

-Acknowledgements

-References (Bibliography followed by webography)

\section{Reviews, commentary, or articles in the Arts and} Social/Human Sciences

\section{- Abstract}

- Scope of the review/article

- The review itself with suitable headings

- Conclusions

- Acknowledgements

- References (Bibliography followed by webography)

\section{FURTHER DETAILS ON STYLE}

Layout: JCAS will contain sections for various colleges of the Academy but will receive and publish articles from non-members as well, subject to editorial review. The following sections will appear in regular numbers of the journal

-Biological Sciences

-Mathematical, Physical and Engineering Sciences

-Arts and Social Sciences
-Opinions/Commentaries

Authors should clearly indicate the section to which their manuscript is directed and should, in addition, indicate whether the work is a Research or a Review Article.

Abstract: All articles should be provided with a brief summary not exceeding 200 words. The abstract should be written in simple language and should highlight the aims of the work, the approach or methodology, the main results obtained and the conclusion reached. Abbreviations should be avoided, and if used, they should be explained at least once. The summary should be presented in English and French.

Introduction or statement of the problem: This section should give the scientific reasons for doing the work and relate the work to previous knowledge and expected outcomes and impact. Only relevant literature should be cited. The introduction should, therefore, not be transformed into a literature review.

Materials and Methods: This section should describe the materials and methods employed in the work in enough detail to enable a qualified reader to duplicate the work. Previously described methods should be cited and not elaborately described. Statistical methods of data treatment should be mentioned if they have been used. If the work involved the use of human subjects, primates, or the release of genetically modified organisms into the environment, it should be stated if ethical clearance from the appropriate authority was obtained. The editor may request a copy of the clearance document or an informed consent form for verification.

Results: This section should describe the results preferably in the same order as predicted by the objectives. Reference should be made to display items such as tables and figures where appropriate. Lengthy discussions should not be undertaken in this section although timely conclusions may be drawn after the presentation of the results.

Discussion: The discussion should highlight what is new in the paper. References should be made to the display items to support the conclusion(s) reached, and 
to previous publications on the same topic. The discussion should be limited to the results described in the manuscript and any implications this may have.

Conclusions: The conclusion(s) should simply be related to the specific objective(s) of the work.

\section{References:}

Bibliography

For a paper with one or two authors the surname(s) is/are referred to in the text. Where the cited paper has more than two co-authors, the surname of the first author is mentioned followed by et al.

Example:

(a) Smith et al. (1997) showed that mosquitoes bite more frequently in the evening than at dawn.

(b) Ngu and Titanji (1981) were the first to describe the use of African patients' own white blood cells for self-treatment of cancer.

(c) For a journal article, the citation is written as follows:

Greenway, P. (1998). Foresight on ageing. Biologist 45, 217-220

(d) For a book, the reference is written giving successively the names(s), the year of publication of the edition of the book consulted or cited, the title of the book, the name of the publisher(s), the city of publication, and the pages cited, e.g : Morris, J. G. (1978). A Biologist's Physical Chemistry. Edward Arnold Ltd. Lon-don

(e) At the end of the manuscript, all the references should be typed in alphabetical order of the first author's surname giving the full title of the article, the journal and pages inclusively;

Webography

Articles from websites and from online journals should be listed in alphabetical order of the surnames of the first authors, followed by the website address (URL) and after the bibliography.

Figures and tables: These should be constructed in such a manner that they can be understood without reading the text. Graphs should preferably be drawn using appropriate computer software and presented on a single page ready for mounting. No writing is allowed on figures directly although the abscissa and ordinates of graphs can be labelled. Appropriate symbols should be used on graphs and explained in the legends.

Legends: Figure and table legends should be typed consecutively on separated sheets at the end of the manuscript. The printer will mount them in the article.

Acknowledgements: These should be presented at the end of the text and before the bibliography. In case of co-authorship of articles, the role of each author should be specified in a brief statement such as "conceived the project, carried out the surveys and drafted the manuscript. etc.

The authors should declare any conflict of interest such as owning stocks in a company whose product has been evaluated and/or tested in the article.

Last revised 19/05/2019 